\title{
New chiral bis(oxazolinyl)bipyridine ligands and application in the iron catalyzed asymmetric hydrosilylation of ketones
}

\author{
Angela Jalba, Guillaume Levitre, Hoda Keipour, Samuel Lauzon, Thierry Ollevier* \\ Département de chimie, Université Laval, 1045 avenue de la Médecine \\ Québec, QC, G1V 0A6, Canada, Fax: +14186567916 \\ thierry.ollevier@chm.ulaval.ca
}

Keywords: chiral bisoxazoline; bipyridine; hydrosilylation; iron; ketones

$C_{2}$ symmetrical 6,6'-bis(oxazolinyl)-2,2'-bipyridine (bipybox) chiral ligands have been synthesized from readily available 2,2'-bipyridine. Catalytic asymmetric hydrosilylation of ketones was studied using this family of ligands in the presence of iron(II) acetate.

\section{Introduction}

The reduction of unsaturated compounds containing $\mathrm{C}=\mathrm{C}, \mathrm{C}=\mathrm{N}$ and $\mathrm{C}=\mathrm{O}$ bonds is among the most studied and probably the most diversified reactions. There is a large variety of methods to perform the reduction of double bonds. ${ }^{[1]}$ Our work focuses on the asymmetric reduction of the $\mathrm{C}=\mathrm{O}$ carbonyl group using the hydrosilylation reaction. ${ }^{[2]}$ There is a large interest to develop procedures affording enantiomerically pure secondary alcohols. Their preparation is essential because they are important building blocks in the synthesis of biologically important products. Especially, chiral secondary alcohols are suitable key intermediates for the synthesis of biologically active components and hence have emerged as important targets in the pharmaceutical industry. ${ }^{[3]}$ Iron is one of the most abundant metals on Earth; it is inexpensive, environmentally benign, and relatively nontoxic in comparison with other metals. From a green chemistry point of view, the development of new Fe-catalyzed methods is of great excitement. ${ }^{[4]}$

Many catalysts are derived from rare metals, and their price or toxicity prevents their use on an industrial scale. Iron, which is ubiquitous, is thus becoming one of the most versatile transition metals. Various reviews have been published in the field of asymmetric catalysis using iron. ${ }^{[1 c, 5]}$

The asymmetric hydrosilylation of ketones was investigated by Nishiyama using $\mathrm{Fe}(\mathrm{OAc})_{2}$ with chiral tridentate bis(oxazoline) ligands, such as pyridine bis(oxazoline) (pybox-bn), or bis(oxazolinephenyl)amine (bopa) and bis(oxazolinyl)phenyl (phebox) ligands. ${ }^{[6]}$ The synthesis and structural characterization of the first chiral iron complexes prepared from 
bis(oxazolinyl)phenyl ligands resulting from the oxidative addition of $\mathrm{Fe}_{2}(\mathrm{CO})_{9}$ to 2-bromosubstituted ligands phebox was also disclosed. ${ }^{[7]}$ The corresponding $\mathrm{Fe}^{\mathrm{II}}$ phebox complex was used in the enantioselective hydrosilylation of ketones. The enantioselectivity of the hydrosilylation of ketones was further improved by ligand design. ${ }^{[8]}$ Bulky substituents on the oxazoline ring led to a higher enantioselectivity (up to $88 \% e e$ ). Gade described the synthesis of well-defined iron complexes containing a bidentate ligand and new enantiopure tridentate $\mathrm{N}-\mathrm{N}-\mathrm{N}$ donor ligands, and used the obtained $\mathrm{Fe}$ complex in the asymmetric hydrosilylation of ketones. ${ }^{[9]}$ More recently, Gade also developed chiral $\mathrm{Fe}^{\mathrm{II}}$ alkyl and $\mathrm{Fe}^{\mathrm{II}}$ alkoxide complexes bearing bis(oxazolinyl-methylidene)isoindoline (boxmi) pincers as stereodirecting ligands, which have been employed as catalysts for enantioselective hydrosilylation reactions with unprecedented activity and selectivity. ${ }^{[10]}$ Chirik studied pybox and box ligands for the hydrosilylation reaction of ketones. ${ }^{[11]}$ Following the same synthetic protocol used for the bis(imino)pyridine $\mathrm{Fe}^{\mathrm{II}}$ dialkyl derivatives, the corresponding pybox and box $\mathrm{Fe}^{\mathrm{II}}$ dialkyl complexes have been also isolated and characterized. Although high conversions were reported for the hydrosilylation of various ketones, the chiral induction of these systems was rather poor (up to $54 \%$ ee). Togni synthesized novel diamine ligands and used them in association with $\mathrm{Fe}(\mathrm{acac})_{2}$ to promote the asymmetric reduction of acetophenone using phenylsilane. ${ }^{[12]}$ Beller developed a highly enantioselective reduction of ketones by selecting $(S, S)$-Meduphos among a series of phosphine ligands. ${ }^{[13]}$ Hunang developed a series of $\mathrm{Fe}^{\mathrm{II}}$ complexes of chiral iminopyridineoxazoline (IPO) ligands. ${ }^{[14]}$ The most sterically hindered $\mathrm{Fe}^{\mathrm{II}}$ catalyst exhibits excellent activity (up to $99 \%$ yield) and high enantioselectivity (up to $93 \%$ ee) in the asymmetric hydrosilylation of aryl ketones.

\section{Results and discussion}

Three novel 6,6'-bis(oxazolinyl)-2,2'bipyridine ligands $\mathbf{1 b - d}$ and already known bipybox-i-Pr 1a ligand were prepared from 2,2'-
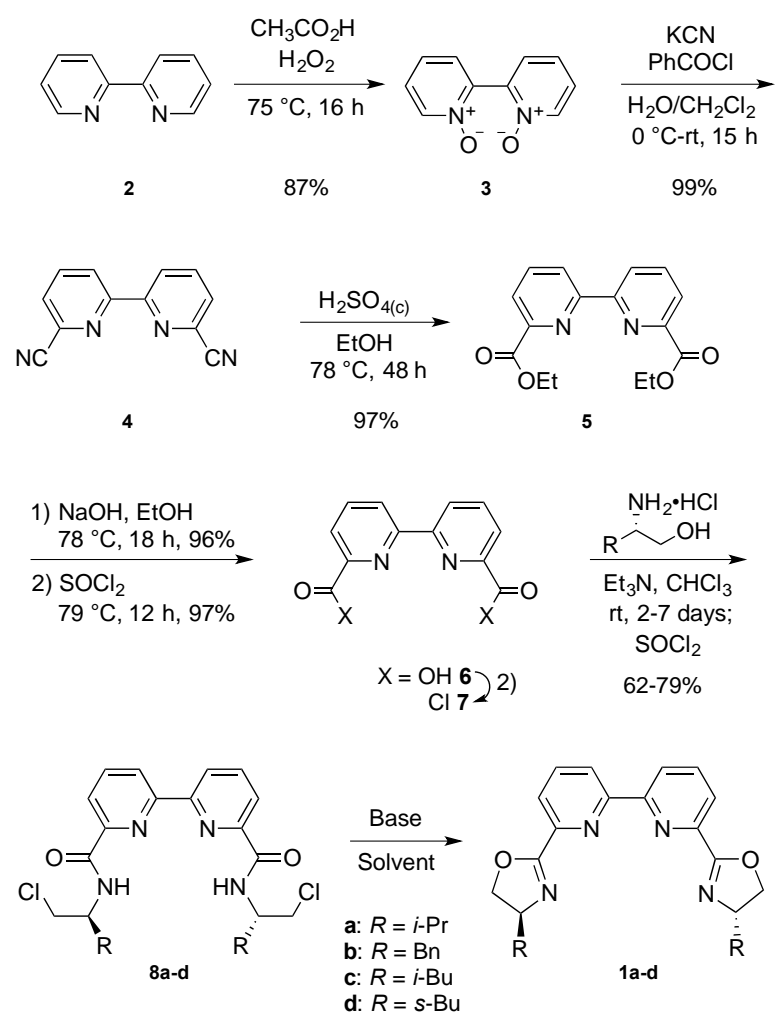

Scheme 1. Synthesis of bipybox ligand

bipyridine $\mathbf{2}$ via a seven-step synthesis 
(Scheme 1). ${ }^{[15]}$ 2,2'-Bipyridine 2 was oxidized to bis- $N$-oxide 3 using hydrogen peroxide in glacial acetic acid. $\alpha$-Bis-cyanation of bis- $N$-oxide 3 with KCN and benzoyl chloride yielded dinitrile $\mathbf{4}$ in excellent yield. ${ }^{[15 c]}$ Conversion of $\mathbf{4}$ into the corresponding $\quad 6,6^{\prime}$-bis(ethoxycarbonyl)-2,2'bipyridine 5 was performed by stirring 4 in ethanol and sulfuric acid. After two days, $\mathbf{5}$ was afforded as a white solid. ${ }^{[15 c]} \mathbf{5}$ was then hydrolyzed in basic conditions to give 6,6'-dicarboxy-2,2'-bipyridine acid 6 in a good yield. The resulting dicarboxylic acid 6 was treated with $\mathrm{SOCl}_{2}$ at reflux to give acid chloride 7 in excellent yield. ${ }^{[15 a]}$ Condensation of 7 with 2 equivalents of various amino alcohols followed after two days by the addition of $\mathrm{SOCl}_{2}$ at room temperature afforded the corresponding bisamidochlorides 8a-d. The previously described cyclization procedure $(\mathrm{NaOH} / \mathrm{MeOH})^{[15 a]}$ was employed successfully to prepare bipybox-i-Pr 1a in an excellent yield (Table 1).

Table 1 Conditions for the synthesis of bipybox ligands

\begin{tabular}{cccccc}
\hline Entry & $\mathrm{R}$ & $\mathrm{Base}$ & Solvent & & $\begin{array}{c}\text { Yield 1 } \\
(\%)\end{array}$ \\
\hline $1^{\mathrm{a}}$ & $i$-Pr & $\mathrm{NaOH}$ & $\mathrm{MeOH}$ & $\mathbf{1 a}$ & 92 \\
$2^{\mathrm{b}}$ & $\mathrm{Bn}$ & $\mathrm{NaOH}$ & $\mathrm{CH}_{2} \mathrm{Cl}_{2}$ & $\mathbf{1 b}$ & 91 \\
$3^{\mathrm{c}}$ & $i$ - $\mathrm{Bu}$ & $\mathrm{NaH}$ & $\mathrm{THF}$ & $\mathbf{1 c}$ & 80 \\
$4^{\mathrm{c}}$ & $s-\mathrm{Bu}$ & $\mathrm{NaH}$ & $\mathrm{THF}$ & $\mathbf{1 d}$ & 85 \\
\hline
\end{tabular}

Condition: a) 8a, $6 \mathrm{~N} \mathrm{NaOH}, \mathrm{MeOH}, 40{ }^{\circ} \mathrm{C}, 2$ days; b) $8 \mathbf{b}, 6 \mathrm{~N}$ $\mathrm{NaOH}, \mathrm{CH}_{2} \mathrm{Cl}_{2}, 40{ }^{\circ} \mathrm{C}, 7$ days; c) $8 \mathbf{c} / \mathbf{8 d}$, $\mathrm{NaH}$ (3.8-4.1 equiv.), THF, rt, 7 days.

Treatment of $\mathbf{8 b - d}$ with $\mathrm{NaOH}$ in $\mathrm{MeOH}$ was ineffective, and the starting bisamidochloride $\mathbf{8 b - d}$ was quantitatively recovered. This is probably explained by the low solubility of 8b-d in methanol. However, bipybox-Bn $\mathbf{1 b}$ was obtained by cyclization of corresponding bis-amidochloride $\mathbf{8 b}$ by using $6 \mathrm{~N} \mathrm{NaOH}$ as a base in $\mathrm{CH}_{2} \mathrm{Cl}_{2}$ giving an excellent yield after recrystallization. The change in the conditions by using the $\mathrm{NaH}$ in THF gave the bipybox-i-Bu 1c, bipybox-s-Bu 1d in a good yield after 7 days at room temperature.

Following our studies in iron catalyzed asymmetric catalysis, ${ }^{[16]}$ we want to disclose our preliminary results using iron(II) acetate conjointly with the aforementioned new series of chiral ligands for the study of the asymmetric hydrosilylation of ketones.

Initially, several chiral bipybox ligands were tested for the reduction of acetophenone 9 to 1-phenylethanol $\mathbf{1 0}$ by using a given set of conditions. Structurally derived bipybox ligands, such as bipybox-i-Pr 1a, bipybox-Bn 1b, bipybox-i-Bu 1c, and bipybox-s-Bu 1d, have been used (Table 2) for the asymmetric hydrosilylation of acetophenone in the presence of $\mathrm{Fe}(\mathrm{OAc})_{2}$ as a catalyst and $\mathrm{PhSiH}_{3}$ as a reducing agent in THF as a solvent to give good to excellent yields of corresponding alcohol $\mathbf{1 0}$ (80-90\%), but poor enantioselectivities. No reaction was observed using either $\mathrm{FeCl}_{2}, \mathrm{FeBr}_{2}$, $\mathrm{Fe}(\mathrm{OTf})_{2}$ or $\mathrm{Fe}\left(\mathrm{ClO}_{4}\right)_{2} \cdot 6 \mathrm{H}_{2} \mathrm{O}$ as a catalyst. Among the salts tested, $\mathrm{Fe}(\mathrm{OAc})_{2}$ was found to be optimal in terms of conversion.

Using these ligands and $\mathrm{Fe}(\mathrm{OAc})_{2}$, different hydride sources, such as $(\mathrm{EtO})_{2} \mathrm{MeSiH}$, $\mathrm{Ph}_{2} \mathrm{SiH}_{2}$ and poly(methylhydroxysilane) 
(PMHS), were surveyed, but $\mathrm{PhSiH}_{3}$ showed better reactivity and selectivity. We also observed a small temperature effect on the selectivity of catalyst. Typically, when the reaction was carried out at room temperature the conversion was slightly lowered and no enantioselectivity was observed. Various organic solvents such as diethyl ether, dichloromethane, and toluene have been studied but the best results have been obtained using THF as solvent.

Table 2 Ligand screening for the asymmetric hydrosilylation of acetophenone using $\mathrm{Fe}(\mathrm{OAc})_{2}$-ligand.

\begin{tabular}{|c|c|c|c|}
\hline $\mathrm{O}$ & $\begin{array}{l}\text { Ligand } \\
\mathrm{Fe}(\mathrm{OAc})_{2} \\
\mathrm{PhSiH}_{3}\end{array}$ & $\mathrm{H}_{3} \mathrm{O}^{+}$ & \\
\hline 9 & $\mathrm{THF}, 65^{\circ} \mathrm{C}, 24 \mathrm{~h}$ & & 10 \\
\hline Entry & Ligand & Yield (\%) & er \\
\hline 1 & bipybox- $i$-Pr 1a & 85 & $40: 60$ \\
\hline 2 & bipybox-Bn $\mathbf{1 b}$ & 90 & $55: 45$ \\
\hline 3 & bipybox-i-Bu 1c & 85 & $52: 48$ \\
\hline 4 & bipybox-s-Bu 1d & 80 & $52: 48$ \\
\hline
\end{tabular}

Conditions: $\mathrm{Fe}(\mathrm{OAc})_{2}$ (5 mol \%), ligand (6 mol \%), acetophenone, $\mathrm{PhSiH}_{3}$ (2 equiv.), THF, $65^{\circ} \mathrm{C}, 24 \mathrm{~h}$.

The pre-catalyst prepared from $\mathrm{FeCl}_{2}$ $(5 \mathrm{~mol} \%)$ and bipybox-i-Pr $1 \mathrm{a}(6 \mathrm{~mol} \%)$ was also tentatively activated using $\mathrm{NaBEt}_{3} \mathrm{H}$ (10 mol $\%) .{ }^{[14]}$ The resulting catalytic system was active for the hydrosilylation of acetophenone 9 using several common silanes. After $24 \mathrm{~h}$, the hydrosilylation product was converted to 1phenylethanol $\mathbf{1 0}$ after acidic work up. $\mathrm{Ph}_{2} \mathrm{SiH}_{2}$ (2 equiv.) and $\mathrm{PhSiH}_{3}$ (2 equiv.) showed good reactivity, giving $75 \%$ and $65 \%$ yield, respectively, but no enantioselectivity was observed. We have also checked the effect of
$\mathrm{AgBF}_{4}(20 \mathrm{~mol} \%)$ as an additive, ${ }^{[15 \mathrm{a}]}$ used in the presence of $\mathrm{FeCl}_{2}(10 \mathrm{~mol} \%)$ and bipybox-i-Pr $1 a$ (12 mol \%), using $\mathrm{Ph}_{2} \mathrm{SiH}_{2}$ (2 equiv.) and $\mathrm{PhSiH}_{3}$ (2 equiv.) as silane sources $\left(65^{\circ} \mathrm{C}, 24 \mathrm{~h}\right)$, but no conversion was observed and the unreacted product was recovered.

\section{Conclusions}

We have presented efficient protocols for the synthesis of three new chiral bis(oxazolinyl)bipyridine ligands starting from $2,2^{\prime}$-bipyridine in seven steps. We have used the newly synthesized ligands, such as bipybox-i-Bu, bipybox-s-Bu, bipybox-Bn and bipybox-i-Pr, in the presence of $\mathrm{Fe}(\mathrm{OAc})_{2}$ as a catalyst in the enantioselective hydrosilylation of acetophenone using $\mathrm{PhSiH}_{3}$ as reducing agent. The enantioselectivities of the reactions were not yet satisfactory using bipybox-i-Bu, bipybox-s-Bu, bipybox-Bn, but it was demonstrated that a promising enantioselectivity was obtained with bipybox-i-Pr ligand used with $\mathrm{Fe}(\mathrm{OAc})_{2}$, together with $\mathrm{PhSiH}_{3}$ as the hydride source, in the hydrosilylation of acetophenone. Our method has the advantage of using a cheap and environmentally benign Lewis acid. The use of these new chiral ligands, complexed in situ with various $\mathrm{Fe}^{\mathrm{II}}$ sources, either under organic or aqueous conditions, is now under investigation for the asymmetric hydrosilylation of various ketones. Further studies to expand the scope and generality of our method are in progress. 


\section{Experimental part}

General information. All chemicals were commercially available. The bipybox ligand 1a was synthesized according to known procedures. ${ }^{[15]}$ The amino alcohols were prepared from corresponding commercially available amino acids by using already known procedures. ${ }^{[17]}$ Solvents (THF, $\mathrm{CH}_{2} \mathrm{Cl}_{2}, \mathrm{Et}_{2} \mathrm{O}$, $\left.\mathrm{CHCl}_{3}\right)$ were distilled prior to use. Thin-layer chromatography (TLC) was carried out on $250 \mu \mathrm{m}$ commercial silica gel plates and compounds were visualized using UV absorbance and/or aqueous $\mathrm{KMnO}_{4}$. Flash column chromatography was performed on silica gel (230-400 mesh). ${ }^{1} \mathrm{H}$ and ${ }^{13} \mathrm{C}$ NMR spectra were recorded on a Varian Inova $400 \mathrm{MHz}$, Varian Inova $500 \mathrm{MHz}$ spectrometer in $\mathrm{CDCl}_{3}$, $\mathrm{D}_{2} \mathrm{O}$ or DMSO- $d_{6}$. For ${ }^{1} \mathrm{H}$ NMR (400 MHz), chemical shift were reported in ppm downfield from tetramethylsilane (TMS) used as internal standard $(\delta=0 \mathrm{ppm})$, and coupling constant and integration (in $\mathrm{Hz}$ ). For ${ }^{13} \mathrm{C}$ NMR $(500 \mathrm{MHz})$, $\mathrm{CDCl}_{3}$ was used as internal standard $(\delta=77.23$ ppm) and spectra were obtained with complete proton decoupling. Chiral HPLC was performed using Daicel Chiracel OD-H chiral column, eluting with $n$-hexanes and $i$-propanol. IR spectra were recorded on a NICOLET 380 FT-IR spectrometer with ZnSn ATR accessory and are reported in reciprocal centimeter $\left(\mathrm{cm}^{-1}\right)$. Melting points (mp) are uncorrected and were recorded on a MEL-TEMP ${ }^{\circledR}$ melting point apparatus.
Reduction of acetophenone. In a glass tube, $\mathrm{Fe}(\mathrm{OAc})_{2}(4.4 \mathrm{mg}, 0.025 \mathrm{mmol})$ and bipybox- $i$ $\operatorname{Pr}(11.4 \mathrm{mg}, 0.03 \mathrm{mmol})$ were added to distilled THF (1 mL). The mixture was stirred for $1 \mathrm{~h}$ in a preheated oil bath at $65{ }^{\circ} \mathrm{C}$ under argon. After removal of the oil bath, acetophenone $(60.1 \mathrm{mg}$, $0.5 \mathrm{mmol})$ and $\mathrm{PhSiH}_{3}(120 \mu \mathrm{L}, 1 \mathrm{mmol})$ were added with a syringe under argon. The mixture was stirred for $20 \mathrm{~h}$ at $65^{\circ} \mathrm{C}$ and the reaction was monitored by TLC; hexane: ethyl acetate (9:1). At $0{ }^{\circ} \mathrm{C}$, aq. $\mathrm{HCl}(2 \mathrm{~N}, 2 \mathrm{~mL})$ was added to quench the reaction. After stirred for $1 \mathrm{~h}$, the mixture was extracted with ethyl acetate $(3 \times 10 \mathrm{~mL})$. The combined organic phases were washed with brine and aq. $\mathrm{NaHCO}_{3}$ and dried over $\mathrm{MgSO}_{4}$. After concentration, the residue was purified by silica gel column chromatography to give the desired alcohol as colorless oil (51.9 mg, 0.43 $\mathrm{mmol}$ ) in a $85 \%$ yield and $20 \%$ ee.

${ }^{1} \mathrm{H}$ NMR (400 MHz, $\left.\mathrm{CDCl}_{3}\right): \delta 7.33-7.40 \mathrm{ppm}$ $(\mathrm{m}, 2 \mathrm{H}) ; 7.25-7.30(\mathrm{~m}, 3 \mathrm{H}), 4.89-4.92(\mathrm{~m}, 1 \mathrm{H})$, $1.49-1.56\left(\mathrm{~d},{ }^{3} J=6.5 \mathrm{~Hz}, 3 \mathrm{H}\right)$. HPLC conditions: Chiralcel OD-H column, $n$-hexane $/ i$-PrOH $=$ 95:5, flow rate $=0.8 \mathrm{~mL} / \mathrm{min}$, wavelength $=254$ $\mathrm{nm}, \mathrm{t}_{R}=9.8 \min (S), \mathrm{t}_{R}=11.6 \min (R)$.

2,2'-Bipyridine- $N, N^{\prime}$-dioxide $\quad(3) .{ }^{[15 b]} \quad \mathrm{A}$ solution of $30 \%$ aqueous hydrogen peroxide (68 $\mathrm{mL}$ ) was added dropwise to 2,2'-bipyridine 2 $(15.1 \mathrm{~g}, 96.0 \mathrm{mmol})$ in glacial acetic acid $(90 \mathrm{~mL})$ at a rate that maintained the temperature between 70 and $80{ }^{\circ} \mathrm{C}$. This mixture was stirred at $75^{\circ} \mathrm{C}$ for $15 \mathrm{~h}$. The colorless solution was then cooled to room temperature, and acetone $(330 \mathrm{~mL})$ was 
added to precipitate the product as a white solid, which was collected by filtration and air-dried (15.8 g, 87\% yield). mp: $298{ }^{\circ} \mathrm{C}$ (lit. 296-298 $\left.{ }^{\circ} \mathrm{C}\right) .{ }^{[18]}{ }^{1} \mathrm{H}$ NMR $\left(400 \mathrm{MHz}, \mathrm{D}_{2} \mathrm{O}\right): \delta 8.27-8.32$ $(\mathrm{m}, 2 \mathrm{H}), 7.68\left(\mathrm{dd},{ }^{3} \mathrm{~J}=8.0,7.3 \mathrm{~Hz}, 2 \mathrm{H}\right), 7.54$ $7.62(\mathrm{~m}, 4 \mathrm{H}) \mathrm{ppm} .{ }^{13} \mathrm{C} \mathrm{NMR}\left(150 \mathrm{MHz}, \mathrm{D}_{2} \mathrm{O}\right): \delta$ 141.68, 139.59, 131.36, 128.77, 128.35 ppm. IR (ZnSn): 3037, 1473, 1426, 1297, $1248 \mathrm{~cm}^{-1}$.

6,6'-Dicyano-2,2'-bipyridine (4). Using a variation of a known procedure, ${ }^{[15 c]}$ 2,2'-bipyridine- $N, N^{\prime}$-dioxide $\quad \mathbf{3} \quad(5.0 \quad \mathrm{~g}$, $26.6 \mathrm{mmol}$ ) and potassium cyanide (10.4 g, 159.3 $\mathrm{mmol})$ were dissolved in water $(53 \mathrm{~mL})$. To this solution cooled at $0{ }^{\circ} \mathrm{C}$, benzoyl chloride $(19.2 \mathrm{~g}$, $136.4 \mathrm{mmol})$ in dichloromethane $(27 \mathrm{~mL})$ were added dropwise. The reaction mixture was stirred for $16 \mathrm{~h}$ at room temperature. The precipitate was filtered, washed with ethanol and dried at room temperature. The product was obtained as a white solid (5.5 g, 99\% yield). mp: $257{ }^{\circ} \mathrm{C}$ (lit. 255 $\left.{ }^{\circ} \mathrm{C}\right) .{ }^{[19]}{ }^{1} \mathrm{H} \mathrm{NMR}\left(400 \mathrm{MHz}, \mathrm{CDCl}_{3}\right): \delta 8.73\left(\mathrm{~d},{ }^{3} \mathrm{~J}\right.$ $=8.0 \mathrm{~Hz}, 2 \mathrm{H}), 8.02\left(\mathrm{dd},{ }^{3} \mathrm{~J}=7.9,7.9 \mathrm{~Hz}, 2 \mathrm{H}\right)$, $7.78\left(\mathrm{~d},{ }^{3} J=7.9 \mathrm{~Hz}, 2 \mathrm{H}\right) \mathrm{ppm} .{ }^{13} \mathrm{C}$ NMR $(150$ $\left.\mathrm{MHz}, \mathrm{CDCl}_{3}\right): \delta 155.51,138.41,133.38,129.09$, 124.66, 117.04 ppm. IR (ZnSn): 1695, 1575, $1433,1156 \mathrm{~cm}^{-1}$.

\section{6,6'-Bis(ethoxycarbonyl)-2,2'-bipyridine}

(5). ${ }^{[15 c]}$ To a solution of $6,6^{\prime}$-dicyano-2,2'bipyridine $4(7.2 \mathrm{~g}, 34.7 \mathrm{mmol})$ in ethanol $(140 \mathrm{~mL})$, concentrated sulfuric acid $(63 \mathrm{~mL})$ was added dropwise. The reaction mixture was heated to reflux for 2 days. The solution was then poured over ice $(30 \mathrm{~g})$ and stirred for $2 \mathrm{~h}$, then extracted with dichloromethane $(3 \mathrm{x})$. The solution was washed with brine, dried over $\mathrm{MgSO}_{4}$ and evaporated to obtain bis(ethoxycarbonyl)-2,2'-bipyridine as a white solid (10.0 g, 97\% yield). mp: $138{ }^{\circ} \mathrm{C}$ (lit. $\left.140-141{ }^{\circ} \mathrm{C}\right) .{ }^{[20]}{ }^{1} \mathrm{H}$ $\operatorname{NMR}\left(400 \mathrm{MHz}, \mathrm{CDCl}_{3}\right): \delta 8.77\left(\mathrm{~d},{ }^{3} J=8.1 \mathrm{~Hz}\right.$, $2 \mathrm{H}), 8.15\left(\mathrm{~d},{ }^{3} \mathrm{~J}=8.1 \mathrm{~Hz}, 2 \mathrm{H}\right), 7.99\left(\mathrm{dd},{ }^{3} J=8.1\right.$, $8.1 \mathrm{~Hz}, 2 \mathrm{H}), 4.50\left(\mathrm{q},{ }^{3} J=7.1 \mathrm{~Hz}, 4 \mathrm{H}\right), 1.48\left(\mathrm{t},{ }^{3} \mathrm{~J}\right.$ $=7.1 \mathrm{~Hz}, 6 \mathrm{H}) \mathrm{ppm} \cdot{ }^{13} \mathrm{C}$ NMR $(150 \mathrm{MHz}$, $\left.\mathrm{CDCl}_{3}\right): \delta 165.16,155.40,147.72,138.00$, 125.38, 124.70, 61.92, $14.31 \mathrm{ppm}$. IR (ZnSn): 2974, 1732, 1699, 1578, $1472 \mathrm{~cm}^{-1}$.

6,6'-Dicarboxy-2,2'-bipyridine acid (6). ${ }^{[15 c]}$ To a solution of 6,6'-bis(ethoxycarbonyl-2,2'bipyridine 5 (5.7 g, $19.0 \mathrm{mmol})$, ethanol $(196 \mathrm{~mL})$ and sodium hydroxide $(3.6 \mathrm{~g}$, $89.0 \mathrm{mmol}$ ) was added. The reaction mixture was heated and refluxed for 1 day, after which the solution was poured into water $(140 \mathrm{~mL})$ and acidified with aqueous $6 \mathrm{~N} \mathrm{HCl}$ until $\mathrm{pH}=1 \sim 2$. The white precipitate was filtered, washed with ethanol and air-dried to afford a white solid ( $4.4 \mathrm{~g}, 96 \%$ yield). mp: $288-289^{\circ} \mathrm{C}$ (lit. dec. 286 $\left.{ }^{\circ} \mathrm{C} ; 288{ }^{\circ} \mathrm{C}\right) .{ }^{[19-20]}{ }^{1} \mathrm{H}$ NMR (400 MHz, DMSO$\left.d_{6}\right): \delta 8.70\left(\mathrm{dd},{ }^{3} \mathrm{~J}=7.7,1.3 \mathrm{~Hz}, 2 \mathrm{H}\right), 8.14\left(\mathrm{dd},{ }^{3} \mathrm{~J}\right.$ $=7.7,7.7 \mathrm{~Hz}, 2 \mathrm{H}), 8.09\left(\mathrm{dd},{ }^{3} J=7.7,1.3 \mathrm{~Hz}, 2 \mathrm{H}\right)$ ppm. ${ }^{13} \mathrm{C}$ NMR (150 MHz, DMSO- $\left.d_{6}\right): \delta 166.30$, 154.82, 148.44, 139.35, 125.67, 124.50 ppm. IR (ZnSn): 2837, 2551, 1690, 1581, $1421 \mathrm{~cm}^{-1}$.

\section{2,2'-Bipyridine-6,6'-dicarbonyl}

chloride

(7). ${ }^{[15 a]} 2,2^{\prime}$-Bipyridine-6,6'-dicarboxylic acid 6 $(6.1 \mathrm{~g}, 24.9 \mathrm{mmol})$ was treated with distilled $\mathrm{SOCl}_{2}(120 \mathrm{~mL})$ at reflux for $17 \mathrm{~h}$. Excess of 
$\mathrm{SOCl}_{2}$ was removed under reduced pressure to afford the acid chloride as a white solid $(6.8 \mathrm{~g}$, $97 \%$ yield). mp: $182-184 \quad{ }^{\circ} \mathrm{C}$ (lit. $177-$ $\left.178{ }^{\circ} \mathrm{C}\right) .{ }^{[20]}{ }^{1} \mathrm{H} \mathrm{NMR}\left(400 \mathrm{MHz}, \mathrm{CDCl}_{3}\right): \delta 8.93$ $\left(\mathrm{d},{ }^{3} J=7.8 \mathrm{~Hz}, 2 \mathrm{H}\right), 8.19\left(\mathrm{~d},{ }^{3} J=7.7 \mathrm{~Hz}, 2 \mathrm{H}\right)$, $8.11\left(\mathrm{dd},{ }^{3} J=7.7,7.7 \mathrm{~Hz}, 2 \mathrm{H}\right) \mathrm{ppm} .{ }^{13} \mathrm{C}$ NMR $\left(150 \mathrm{MHz}, \mathrm{CDCl}_{3}\right): \delta 216.70,155.14,148.25$, 138.77, 126.31, 125.78 ppm. IR (ZnSn): 3201, $2733,1728,1613,1428 \mathrm{~cm}^{-1}$.

General procedure to prepare 2,2'-bipyridine6,6'-dicarboxamide (8). ${ }^{[15 a]}$ To a solution of amino alcohol $(5.34 \mathrm{mmol})$ and triethylamine (1.5 mL, $10.7 \mathrm{mmol})$ in distilled $\mathrm{CHCl}_{3}(15 \mathrm{~mL})$, the acid chloride 7 (0.5 g, $1.8 \mathrm{mmol})$ was added at $0{ }^{\circ} \mathrm{C}$. The mixture was stirred for 2 days at room temperature and then distilled $\mathrm{SOCl}_{2}$ (3.4 $\mathrm{mL}$ ) was added dropwise. After stirring for $12 \mathrm{~h}$ excess of $\mathrm{SOCl}_{2}$ was removed under reduced pressure to give a black oil, which was extracted with $\mathrm{CH}_{2} \mathrm{Cl}_{2}(3 \times 80 \mathrm{~mL})$. The combined organic phases were washed with brine and dried over $\mathrm{MgSO}_{4}$. Concentration gave black solid product which was purified by silica gel column to give the product $\left(\mathrm{CH}_{2} \mathrm{Cl}_{2} / \mathrm{MeOH}=98: 2\right)$.

\section{N6,N6'-bis((S)-1-chloro-3-methylbutan-2-yl)-}

2,2'-bipyridine-6,6'-dicarboxamide $\quad(8 a) .{ }^{[15 a]}$

White solid (4.6 g, 78\% yield). mp $210{ }^{\circ} \mathrm{C}$ (lit. $\left.210{ }^{\circ} \mathrm{C}\right) .\left[{ }^{[15 a]}{ }^{1} \mathrm{H}\right.$ NMR $\left(400 \mathrm{MHz}, \mathrm{CDCl}_{3}\right): \delta 8.52$ $\left(\mathrm{d},{ }^{3} J=7.8 \mathrm{~Hz}, 2 \mathrm{H}\right), 8.35\left(\mathrm{~d},{ }^{3} J=9.4 \mathrm{~Hz}, 2 \mathrm{H}\right)$, $8.28\left(\mathrm{~d},{ }^{3} \mathrm{~J}=7.7 \mathrm{~Hz}, 2 \mathrm{H}\right), 8.06\left(\mathrm{dd},{ }^{3} J=7.8,7.8\right.$ $\mathrm{Hz}, 2 \mathrm{H}$ ), 4.17-4.26 (m, 2H), 3.89 (dd, ${ }^{2} J=11.3$ $\left.\mathrm{Hz},{ }^{3} J=3.7 \mathrm{~Hz} 2 \mathrm{H}\right), 3.80\left(\mathrm{dd},{ }^{2} J=11.3 \mathrm{~Hz},{ }^{3} J=\right.$
$3.7 \mathrm{~Hz}, 2 \mathrm{H}), 2.12-2.24(\mathrm{~m}, 2 \mathrm{H}), 1.09\left(\mathrm{~d},{ }^{3} J=6.8\right.$ $\mathrm{Hz}, 6 \mathrm{H}), 1.06\left(\mathrm{~d},{ }^{3} \mathrm{~J}=6.8 \mathrm{~Hz}, 6 \mathrm{H}\right) .{ }^{13} \mathrm{C}$ NMR $(150$ $\left.\mathrm{MHz}, \mathrm{CDCl}_{3}\right): \delta 163.82,153.68,149.18,138.72$, $123.57,122.89,55.02,46.84,29.56,19.46,18.89$ ppm. IR (ZnSn): 3279, 2961, 1655, 1579, 1562, $1517,1469 \mathrm{~cm}^{-1}$.

N6,N6'-bis((S)-1-chloro-3-phenylpropan-2yl)-2,2'-bipyridine-6,6'-dicarboxamide $(8 \mathrm{~b})$. Yellow solid (0.8 g, 79\% yield). mp: 188 $190{ }^{\circ} \mathrm{C} .{ }^{1} \mathrm{H}$ NMR $\left(400 \mathrm{MHz}, \mathrm{CDCl}_{3}\right): \delta 8.49(\mathrm{~d}$, $\left.{ }^{3} J=7.8 \mathrm{~Hz}, 2 \mathrm{H}\right), 8.43\left(\mathrm{~d},{ }^{3} J=8.9 \mathrm{~Hz}, 2 \mathrm{H}\right), 8.28$ $\left(\mathrm{d},{ }^{3} J=7.6 \mathrm{~Hz}, 2 \mathrm{H}\right), 8.07\left(\mathrm{dd},{ }^{3} J=7.7,7.7 \mathrm{~Hz}\right.$, 2H), 7.34-7.39 (m, 8H), 7.26-7.33 (m, 2H), 4.66-4.76 (m, 2H), $3.76\left(\mathrm{dd},{ }^{2} J=11.1 \mathrm{~Hz}\right.$, $\left.{ }^{3} J=4.9 \mathrm{~Hz}, 2 \mathrm{H}\right), 3.69\left(\mathrm{dd},{ }^{2} J=11.2 \mathrm{~Hz},{ }^{3} J=3.5\right.$ $\mathrm{Hz}, 2 \mathrm{H}), 3.20\left(\mathrm{dd},{ }^{2} J=13.8 \mathrm{~Hz},{ }^{3} J=5.6 \mathrm{~Hz}, 2 \mathrm{H}\right)$, $3.09\left(\mathrm{dd},{ }^{2} J=13.8 \mathrm{~Hz},{ }^{3} J=8.2 \mathrm{~Hz}, 2 \mathrm{H}\right) \mathrm{ppm} .{ }^{13} \mathrm{C}$ NMR (150 MHz, $\left.\mathrm{CDCl}_{3}\right): \delta 163.49,153.57$, 149.07, 138.65, 136.69, 129.51, 128.84, 127.03, 123.64, 122.83, 50.58, 46.27, 37.39 ppm. IR (ZnSn): 3293, 3028, 1734, 1650. 1579, 1517, $1454 \mathrm{~cm}^{-1}$.

\section{N6,N6'-bis( $(S)$-1-chloro-4-methylpentan-2-} yl)-2,2'-bipyridine-6,6'-dicarboxamide (8c). White solid (1.7 g, 78\% yield). mp: $184-185^{\circ} \mathrm{C}$. ${ }^{1} \mathrm{H}$ NMR (400 MHz, $\left.\mathrm{CDCl}_{3}\right): \delta 8.53\left(\mathrm{dd},{ }^{3} J=7.9\right.$, $1.1 \mathrm{~Hz}, 2 \mathrm{H}), 8.27\left(\mathrm{dd},{ }^{3} \mathrm{~J}=7.7,1.1 \mathrm{~Hz}, 2 \mathrm{H}\right), 8.24$ $\left(\mathrm{d},{ }^{3} J=9.1 \mathrm{~Hz}, 2 \mathrm{H}\right), 8.06\left(\mathrm{dd},{ }^{3} J=7.8,7.8 \mathrm{~Hz}\right.$, $2 \mathrm{H}), 4.55-4.63(\mathrm{~m}, 2 \mathrm{H}), 3.87\left(\mathrm{dd},{ }^{2} \mathrm{~J}=11.1 \mathrm{~Hz}\right.$, $\left.{ }^{3} J=3.6 \mathrm{~Hz}, 2 \mathrm{H}\right), 3.76\left(\mathrm{dd},{ }^{2} J=11.2 \mathrm{~Hz},{ }^{3} J=3.6\right.$ $\mathrm{Hz}, 2 \mathrm{H}), 1.68-1.77$ (m, 4H), 1.56-1.68 (m, 2H), $1.01\left(\mathrm{~d},{ }^{3} \mathrm{~J}=6.3 \mathrm{~Hz}, 6 \mathrm{H}\right), 0.99\left(\mathrm{~d},{ }^{3} J=6.2 \mathrm{~Hz}\right.$, 
$6 \mathrm{H})$ ppm. ${ }^{13} \mathrm{C}$ NMR $\left(150 \mathrm{MHz}, \mathrm{CDCl}_{3}\right): \delta$ 163.56, 153.62, 138.61, 123.60, 122.85, 122.79, 48.75, 47.65, 41.06, 24.88, 22.84, 22.38 ppm. IR (ZnSn): 3305, 2954, 1722, 1650, 1566, $1465 \mathrm{~cm}^{-}$ 1 .

\section{N6,N6'-bis((2S,3R)-1-chloro-3-methylpentan-}

2-yl)-2,2'-bipyridine-6,6'-dicarboxamide (8d).

Yellow solid (0.5 g, 62\% yield). mp: $148{ }^{\circ} \mathrm{C}$. ${ }^{1} \mathrm{H} \quad \mathrm{NMR} \quad\left(400 \mathrm{MHz}, \mathrm{CDCl}_{3}\right): \quad \delta 8.53 \quad(\mathrm{~d}$, $\left.{ }^{3} J=7.9 \mathrm{~Hz}, 2 \mathrm{H}\right), 8.35\left(\mathrm{~d},{ }^{3} J=9.8 \mathrm{~Hz}, 2 \mathrm{H}\right), 8.28$ $\left(\mathrm{d},{ }^{3} J=7.7 \mathrm{~Hz}, 2 \mathrm{H}\right), 8.06\left(\mathrm{dd},{ }^{3} J=7.8,7.8 \mathrm{~Hz}\right.$, 2H), 4.23-4.33 (m, 2H), $3.90\left(\mathrm{dd},{ }^{2} J=11.5 \mathrm{~Hz}\right.$, $\left.{ }^{3} J=3.8 \mathrm{~Hz}, 2 \mathrm{H}\right), 3.83\left(\mathrm{dd},{ }^{2} J=11.5 \mathrm{~Hz},{ }^{3} J=3.8\right.$ $\mathrm{Hz}, 2 \mathrm{H}), 1.85-2.03$ (m, 2H), 1.56-1.71 (m, 2H), $1.18-1.35(\mathrm{~m}, 2 \mathrm{H}), 1.05\left(\mathrm{~d},{ }^{3} \mathrm{~J}=6.7 \mathrm{~Hz}, 6 \mathrm{H}\right), 0.95$ $\left(\mathrm{t},{ }^{3} J=7.4 \mathrm{~Hz}, 6 \mathrm{H}\right) \mathrm{ppm} .{ }^{13} \mathrm{C} \mathrm{NMR}(150 \mathrm{MHz}$, $\left.\mathrm{CDCl}_{3}\right): \delta 163.66,153.56,149.10,138.61$, 123.50, 122.77, 53.61, 46.95, 35.77, 25.17, 15.38, 11.04 ppm. IR (ZnSn): 3313, 2962, 2874, 1722, 1652, 1579, $1462 \mathrm{~cm}^{-1}$.

6,6'-Bis(4-(S)-isopropyloxazolin-2-y1)-2,2'-bipyridine (1a). ${ }^{[15 a]}$ To a solution of the N6,N6'bis((S)-1-chloro-3-methylbutan-2-yl)-2,2'-bipyridine-6,6'-dicarboxamide $\mathbf{8 a}$ ( $2 \mathrm{~g}, 4.4 \mathrm{mmol})$ in $\mathrm{MeOH}(110 \mathrm{~mL})$, aqueous $\mathrm{NaOH}(6 \mathrm{~N}, 35 \mathrm{~mL})$ was added. The mixture was then stirred 2 days at $40{ }^{\circ} \mathrm{C}$. The reaction was monitored by TLC $\left(\mathrm{CH}_{2} \mathrm{Cl}_{2} / \mathrm{MeOH} 9: 1\right)$. The mixture was extracted with $\mathrm{CH}_{2} \mathrm{Cl}_{2}$, dried over $\mathrm{MgSO}_{4}$, and concentrated in vacuo to give a white solid. After recrystallization (ethyl acetate/hexane) the product was obtained as a white solid (1.6 g, 96\% yield). mp: $184{ }^{\circ} \mathrm{C}$ (lit. $\left.187{ }^{\circ} \mathrm{C}\right) .[15 \mathrm{a}]{ }^{1} \mathrm{H}$ NMR $\left(500 \mathrm{MHz}, \mathrm{CDCl}_{3}\right) \delta 8.66\left(\mathrm{dd},{ }^{3} J=7.9,1.1 \mathrm{~Hz}\right.$, 2H), $8.12\left(\mathrm{dd},{ }^{3} J=7.8,1.1 \mathrm{~Hz}, 2 \mathrm{H}\right), 7.91\left(\mathrm{dd},{ }^{3} J\right.$ $=7.87 .8 \mathrm{~Hz}, 2 \mathrm{H}), 4.55\left(\mathrm{dd},{ }^{3} J=9.6 \mathrm{~Hz},{ }^{2} J=8.2\right.$ $\mathrm{Hz}, 2 \mathrm{H}), 4.22\left(\mathrm{dd},{ }^{3} J=8.2 \mathrm{~Hz},{ }^{2} J=8.2 \mathrm{~Hz}, 2 \mathrm{H}\right)$, 4.16-4.22 (m, 2H), 1.88-1.96 (m, 2H), 1.09 (d, $\left.{ }^{3} J=6.8 \mathrm{~Hz}, 6 \mathrm{H}\right), 0.97\left(\mathrm{~d},{ }^{3} J=6.8 \mathrm{~Hz}, 6 \mathrm{H}\right) \mathrm{ppm}$. ${ }^{13} \mathrm{C} \mathrm{NMR}\left(150 \mathrm{MHz}, \mathrm{CDCl}_{3}\right) \delta 162.66,155.34$, 146.27, 137.53, 124.38, 123.53, 72.97, 70.79, 32.79, 19.12, 18.19 ppm. IR (ZnSn): 2953, 2864, $1636,1577,1474,1445,1263 \mathrm{~cm}^{-1}$.

\section{6,6'-Bis(4-(S)-benzyloxazolin-2-y1)-2,2'-bi-}

pyridine (1b). To a solution of the N6,N6'bis((S)-1-chloro-3-phenylpropan-2-yl)-2,2'-bi-

pyridine-6,6'-dicarboxamide $\quad \mathbf{8 b} \quad(0.3 \quad \mathrm{~g}$, $0.6 \mathrm{mmol})$ in $\mathrm{CH}_{2} \mathrm{Cl}_{2}(5.5 \mathrm{~mL})$, aqueous $\mathrm{NaOH}$ $(6 \mathrm{~N}, 3.4 \mathrm{~mL})$ was added. The mixture was stirred 7 days at $40{ }^{\circ} \mathrm{C}$. The reaction was monitored by TLC $\left(\mathrm{CH}_{2} \mathrm{Cl}_{2} / \mathrm{MeOH}\right.$ 9:1). The mixture was extracted with $\mathrm{CH}_{2} \mathrm{Cl}_{2}$, dried over $\mathrm{MgSO}_{4}$, and concentrated in vacuo to give a brown solid. After recrystallization (ethyl acetate/hexane) the product was obtained as a yellow solid $(1.5 \mathrm{~g}$, 91\% yield). mp: $206-208{ }^{\circ} \mathrm{C} .{ }^{1} \mathrm{H}$ NMR (500 $\left.\mathrm{MHz} \mathrm{CDCl}_{3}\right): \delta 8.68\left(\mathrm{dd},{ }^{3} \mathrm{~J}=7.8,1.1 \mathrm{~Hz}, 2 \mathrm{H}\right)$, $8.12\left(\mathrm{dd},{ }^{3} J=7.8,1.1 \mathrm{~Hz}, 2 \mathrm{H}\right), 7.94\left(\mathrm{dd},{ }^{3} J=7.8\right.$, $7.8 \mathrm{~Hz}, 2 \mathrm{H}), 7.32-7.36(\mathrm{~m}, 4 \mathrm{H}), 7.24-7.30$ (m, 6H), 4.66-4.75 (m, 2H), $4.49\left(\mathrm{dd},{ }^{3} J=9.4 \mathrm{~Hz},{ }^{2} J\right.$ $=8.5 \mathrm{~Hz}, 2 \mathrm{H}), 4.29\left(\mathrm{dd},{ }^{2} J=8.5 \mathrm{~Hz},{ }^{3} J=7.6 \mathrm{~Hz}\right.$, $2 \mathrm{H}), 3.35\left(\mathrm{dd},{ }^{2} J=13.8 \mathrm{~Hz},{ }^{3} J=5.0 \mathrm{~Hz}, 2 \mathrm{H}\right)$, $2.80\left(\mathrm{dd},{ }^{2} J=13.8 \mathrm{~Hz},{ }^{3} J=9.2 \mathrm{~Hz}, 2 \mathrm{H}\right) \mathrm{ppm} .{ }^{13} \mathrm{C}$ NMR (100 MHz, $\left.\mathrm{CDCl}_{3}\right): \delta 163.12,155.34$, 146.17, 137.76, 137.62, 129.17, 128.54, 126.53, 124.40, 123.55, 72.39, 68.09, 41.64 ppm. IR 
(ZnSn): 3274, 2884, 1657, 1577, 1444, $1368 \mathrm{~cm}^{-}$ 1 .

\section{6,6'-Bis(4-(S)-isobutyloxazolin-2-y1)-2,2' -bi-} pyridine (1c). To a solution of the N6,N6'bis((S)-1-chloro-4-methylpentan-2-yl)-2,2'-bipyridine-6,6'-dicarboxamide $8 \mathbf{c}(0.5 \mathrm{~g}, 1.1 \mathrm{mmol})$ in THF (11 mL, $0.1 \mathrm{M}), \mathrm{NaH}(0.1 \mathrm{~g}, 4.24 \mathrm{mmol})$ was added. The mixture was then stirred 7 days at room temperature under argon atmosphere. The reaction was monitored by TLC $\left(\mathrm{CH}_{2} \mathrm{Cl}_{2} / \mathrm{MeOH} 9: 1\right)$. The mixture was extracted with $\mathrm{CH}_{2} \mathrm{Cl}_{2}$, dried over $\mathrm{MgSO}_{4}$, to afford a brownish solid. After recrystallization (ethyl acetate/hexane) the product was obtained as a white solid (0.35 g, 80\% yield). mp: $134{ }^{\circ} \mathrm{C} .{ }^{1} \mathrm{H}$ NMR (400 MHz, $\left.\mathrm{CDCl}_{3}\right): \delta 8.67\left(\mathrm{~d},{ }^{3} J=7.9 \mathrm{~Hz}\right.$, $2 \mathrm{H}), 8.10\left(\mathrm{dd},{ }^{3} J=7.8,1.1 \mathrm{~Hz}, 2 \mathrm{H}\right), 7.92\left(\mathrm{dd},{ }^{3} J\right.$ $=7.8,7.8 \mathrm{~Hz}, 2 \mathrm{H}), 4.65\left(\mathrm{dd},{ }^{3} \mathrm{~J}=9.5 \mathrm{~Hz},{ }^{2} \mathrm{~J}=8.1\right.$ $\mathrm{Hz}, 2 \mathrm{H}), 4.41-4.48(\mathrm{~m}, 2 \mathrm{H}), 4.14\left(\mathrm{dd},{ }^{3} \mathrm{~J}=8.1\right.$ $\left.\mathrm{Hz},{ }^{2} J=8.1 \mathrm{~Hz}, 2 \mathrm{H}\right), 1.84-1.92(\mathrm{~m}, 2 \mathrm{H}), 1.78$ $1.84(\mathrm{~m}, 2 \mathrm{H}), 1.42-1.48(\mathrm{~m}, 2 \mathrm{H}), 1.02\left(\mathrm{~d},{ }^{3} \mathrm{~J}=6.6\right.$ $\mathrm{Hz}, 6 \mathrm{H}), 1.00\left(\mathrm{~d},{ }^{3} J=6.5 \mathrm{~Hz}, 6 \mathrm{H}\right) .{ }^{13} \mathrm{C}$ NMR $(100$ $\left.\mathrm{MHz}, \mathrm{CDCl}_{3}\right): \delta 162.08,154.95,146.06,137.10$, 124.00, 123.02, 73.22, 65.09, 45.15, 25.13, 22.82, 22.61 ppm. IR (ZnSn): 2953, 2924, 1632, $1577,13801279 \mathrm{~cm}^{-1}$.

\section{6,6'-Bis(4-(S)-secbutyloxazolin-2-y1)-2,2' -bi-}

pyridine (1d). To a solution of the N6,N6'bis((2S,3R)-1-chloro-3-methylpentan-2-yl)-2,2'bipyridine-6,6'-dicarboxamide $\quad \mathbf{8 d} \quad(1.4 \mathrm{~g}$, $2.9 \mathrm{mmol})$ in THF (30 mL, $0.1 \mathrm{M}), \mathrm{NaH}(0.3 \mathrm{~g}$, $11.9 \mathrm{mmol}$ ) was added. The mixture was stirred 7 days at room temperature under argon atmosphere. The reaction was monitored by TLC $\left(\mathrm{CH}_{2} \mathrm{Cl}_{2} / \mathrm{MeOH} 9: 1\right)$. The mixture was extracted with $\mathrm{CH}_{2} \mathrm{Cl}_{2}$, dried over $\mathrm{MgSO}_{4}$, and concentrated to give a brownish solid. After recrystallization (ethyl acetate/hexane) the product was obtained as a white solid (1.0 g, 85\% yield). mp: ${ }^{150-152}{ }^{\circ} \mathrm{C} .{ }^{1} \mathrm{H}$ NMR (500 MHz, $\left.\mathrm{CDCl}_{3}\right): \delta 8.66\left(\mathrm{~d},{ }^{3} J=7.9 \mathrm{~Hz}, 2 \mathrm{H}\right), 8.12\left(\mathrm{dd},{ }^{3} J\right.$ $=7.7,1.1 \mathrm{~Hz}, 2 \mathrm{H}), 7.91\left(\mathrm{dd},{ }^{3} J=7.8,7.8 \mathrm{~Hz}, 2 \mathrm{H}\right)$, $4.54\left(\mathrm{dd},{ }^{3} \mathrm{~J}=9.3 \mathrm{~Hz},{ }^{2} \mathrm{~J}=8.0 \mathrm{~Hz}, 2 \mathrm{H}\right), 4.30-4.36$ $(\mathrm{m}, 2 \mathrm{H}), 4.28\left(\mathrm{dd},{ }^{3} J=8.2 \mathrm{~Hz},{ }^{2} J=8.0 \mathrm{~Hz}, 2 \mathrm{H}\right)$, 1.75-1.85 (m, 2H), 1.65-1.74 (m, 2H), 1.24-1.35 (m, 2H), $0.99\left(\mathrm{t},{ }^{3} J=7.5 \mathrm{~Hz}, 6 \mathrm{H}\right), 0.92\left(\mathrm{~d},{ }^{3} J=\right.$ $6.8 \mathrm{~Hz}, 6 \mathrm{H}) \mathrm{ppm} .{ }^{13} \mathrm{C} \mathrm{NMR}\left(100 \mathrm{MHz}, \mathrm{CDCl}_{3}\right)$ : $\delta 162.39,155.18,146.23,137.33,124.22$, 123.30, 71.33, 70.13, 38.95, 26.03, 14.30, 11.41 ppm. IR (ZnSn): 2959, 2876, 1657, 1577, 1445, $1381 \mathrm{~cm}^{-1}$.

\section{Acknowledgements}

We are grateful to the Natural Sciences and Engineering Research Council of Canada (NSERC), the Centre in Green Chemistry and Catalysis (CGCC) and Université Laval for financial support of our program. H. K. thanks CGCC for a scholarship.

\section{References}

[1] a) R. Malacea, R. Poli, E. Manoury, Coord. Chem. Rev. 2010, 254, 729-752; b) S. Gaillard, J. L. Renaud, ChemSusChem 2008, 1, 505-509; c) M. Darwish, M. Wills, Catal. Sci. Technol. 2012, 2, 243-255.

a) H. Nishiyama, in Comprehensive Chirality, Vol. 5, Elsevier Ltd., 2012, pp. 318-333; b) M. Zhang, A. Zhang, Appl. Organomet. Chem. 2010, 24, 751-757; c) A. K. Roy, in Adv. Organomet. Chem., Vol. 55, Elsevier, 2007, pp. 1-59. 
[3] a) J. N. Zhou, Q. Fang, Y. H. Hu, L. Y. Yang, F. F. Wu, L. J. Xie, J. Wu, S. Li, Org. Biomol. Chem. 2014, 12, 1009-1017; b) S. B. Qi, M. Li, S. Li, J. N. Zhou, J. W. Wu, F. Yu, X. C. Zhang, A. S. Chan, J. Wu, Org. Biomol. Chem. 2013, 11, 929937; c) Y. Z. Sui, X. C. Zhang, J. W. Wu, S. Li, J. N. Zhou, M. Li, W. Fang, A. S. Chan, J. Wu, Chem. Eur. J. 2012, 18, 7486-7492; d) F. Yu, J. N. Zhou, X. C. Zhang, Y. Z. Sui, F. F. Wu, L. J. Xie, A. S. Chan, J. Wu, Chem. Eur. J. 2011, 17, 14234-14240.

[4] a) I. Bauer, H.-J. Knoelker, Chem. Rev. 2015, 115, 3170-3387; b) C. Bolm, J. Legros, J. Le Paih, L. Zani, Chem. Rev. 2004, 104, 6217-6254; c) S. Enthaler, K. Junge, M. Beller, Angew. Chem. Int. Ed. 2008, 47, 3317-3321; d) L. C. Misal Castro, H. Li, J.-B. Sortais, C. Darcel, Green Chem. 2015, 17, 2283-2303; e) E. Bauer, ed., Iron catalysis II, Springer, Heidelberg, 2015.

[5] a) T. Ollevier, Catal. Sci. Technol. 2016, doi: 10.1039/C5CY01357G; b) K. Gopalaiah, Chem. Rev. 2013, 113, 3248-3296; c) R. H. Morris, Chem. Soc. Rev. 2009, 38, 2282-2291; d) P. E. Sues, K. Z. Demmans, R. H. Morris, Dalton Trans. 2014, 43, 7650-7667; e) A. Fingerhut, O. V. Serdyuk, S. B. Tsogoeva, Green Chem. 2015, 17, 2042-2058; f) T. Ollevier, H. Keipour, in Topics in Organometallic Chemistry (E. Bauer ed.), Springer, Heidelberg, 2015; g) S. Enthaler, in Comprehensive Inorganic Chemistry II, 2013, pp. 549-562.

[6] a) H. Nishiyama, A. Furuta, Chem. Commun. 2007, 760-762; b) T. Inagaki, A. Ito, J.-i. Ito, H. Nishiyama, Angew. Chem. Int. Ed. 2010, 49, 9384-9387.

[7] S. Hosokawa, J.-i. Ito, H. Nishiyama, Organometallics 2010, 29, 5773-5775.

[8] T. Inagaki, L. T. Phong, A. Furuta, J.-i. Ito, H. Nishiyama, Chem. Eur. J. 2010, 16, 3090-3096.

[9] B. K. Langlotz, H. Wadepohl, L. H. Gade, Angew. Chem. Int. Ed. 2008, 47, 4670-4674.

[10] T. Bleith, H. Wadepohl, L. H. Gade, J. Am. Chem. Soc. 2015, 137, 2456-2459.

[11] A. M. Tondreau, J. M. Darmon, B. M. Wile, S. K. Floyd, E. Lobkovsky, P. J. Chirik, Organometallics 2009, 28, 3928-3940.

[12] M. Flueckiger, A. Togni, Eur. J. Org. Chem. 2011, 4353-4360.

[13] N. S. Shaikh, S. Enthaler, K. Junge, M. Beller, Angew. Chem., Int. Ed. 2008, 47, 2497-2501.

[14] Z. Zuo, L. Zhang, X. Leng, Z. Huang, Chem. Commun. 2015, 51, 5073-5076.

[15] a) H. Nishiyama, S. Yamaguchi, S.-B. Park, K. Itoh, Tetrahedron: Asymmetry 1993, 4, 143-150; b) D. Zhang, J. P. Telo, L. Chen, S. E. Hightower, E. L. Clennan, J. Phys. Chem. A 2007, 111, 13567-13574; c) W. Sharmoukh, N. K. Allam, ACS Appl. Mater. Interfaces 2012, 4, 4413-4418.

[16] a) T. Ollevier, B. Plancq, Chem. Commun. 2012, 48, 2289-2291; b) B. Plancq, T. Ollevier, Chem.
Commun. 2012, 48, 3806-3808; c) M. Lafantaisie, A. Mirabaud, B. Plancq, T. Ollevier, ChemCatChem 2014, 6, 2244-2247; d) B. Plancq, M. Lafantaisie, S. Companys, C. Maroun, T. Ollevier, Org. Biomol. Chem. 2013, 11, 74637466.

[17] S. Lou, G. C. Fu, Org. Synth. 2010, 87, 310-316.

[18] C. B. Aakeroey, T. K. Wijethunga, J. Desper, CrystEngComm 2014, 16, 28-31.

[19] F. H. Burstall, J. Chem. Soc. 1938, 1662-1672.

[20] E. Buhleier, W. Wehner, F. Voegtle, Chem. Ber. 1978, 111, 200-204. 